\title{
The Permanent Challenge of Treating Diabetic Patients with Complex Coronary Artery Disease
}

\author{
Áurea J. Chaves
}

D iabetes mellitus is a known factor that influences coronary artery bypass graft (CABG) surgery effectiveness. Selecting the most appropriate intervention, whether surgical or percutaneous, is essential to provide the best care for patients with multivessel coronary disease. Since the mid-1990s, with the publication of the BARI trial results, diabetes has become an important variable to be considered in the decision-making of clinicians, interventional physicians, and cardiac surgeons. Recently, we have witnessed a series of studies comparing surgical procedures with more contemporary percutaneous interventions, whose results indicated comparable mortality between the two strategies. The results of the most recent studies, which employ drug-eluting stents, have been regarded with caution, as the data are derived from randomized trials without statistical power or subgroup analysis.

The randomized trial FREEDOM, with 1,900 patients, presented in November 2012 at the Scientific Sessions of American Heart Association, was designed to answer the question of which revascularization strategy would be the best in diabetic patients with complex multivessel disease. The trial's long-awaited results are discussed in depth in an editorial by Centemero, Abizaid, and Sousa, of Instituto Dante Pazzanese de Cardiologia (São Paulo, SP, Brazil). The authors highlight the many virtues of this study, as well as its limitations, and conclude by reinforcing the need to individualize decisions, and for the "heart team" to discuss therapeutic possibilities, involving the patient and family in the final decision.

This edition of Revista Brasileira de Cardiologia Invasiva (RBCl) presents numerous studies that address issues of significant current interest. Costa Jr. et al., from Hospital do Coração da Associação do Sanatório Sírio (São Paulo, SP, Brazil), present another contribution from the DESIRE registry, this time evaluating the changes occurring in the last ten years regarding the indication and performance of percutaneous coronary intervention $(\mathrm{PCl})$ with drug-eluting stents. The authors describe the evolution of $\mathrm{PCl}$ as the prevalent mode of revascularization compared with surgery at that institution; the diffusion of drug-eluting stents, which reached almost $90 \%$ of the procedures last year; the increasing angiographic complexity evaluated by the SYNTAX score; and the good clinical outcomes in the five-year follow-up of over $98 \%$ of that population.

Andrade et al., from Santa Casa de Marília (Marília, $\mathrm{SP}$, Brazil), address the issue of inter-hospital transfer for primary $\mathrm{PCl}$ in their city, which has an integrated and efficient healthcare system for cardiovascular emergencies. The authors evaluate the efficacy and the safety outcomes of patients admitted or transferred to undergo the procedure. The researchers complement their analysis with very up-to-date discussions of the matter, addressing the issue of patient transfer in Brazil, which experiences the well-known problems related to urban traffic.

Aguiar Filho et al., from Instituto Dante Pazzanese de Cardiologia, address the incorporation of transradial $\mathrm{PCl}$ into their center, which is known for training interventional cardiologists. The authors demonstrate the increasingly frequent use of this access route over the last few years compared to the traditional femoral approach, and report the profiles of selected patients. The researchers demonstrate that the clinical outcomes can be similar to the outcomes in the literature, even when these procedures are performed by physicians in training, supervised by the interventional cardiologists in charge.

Farinazzo et al., from Hospital Bandeirantes (São Paulo, SP, Brazil), explore the results of ad hoc $\mathrm{PCl}$, a subject that is rarely explored in the Brazilian literature, in a large registry from that institution. The authors report the percentage of the population that underwent this intervention; the method's clinical, angiographic, and procedural characterization; and in-hospital clinical outcomes compared with the outcomes in patients who underwent scheduled interventions. In their discussion, the researchers recall the limits of the indication of ad hoc $\mathrm{PCl}$, according to the recent publication of a consensus by Society for Cardiovascular Angiography and Interventions (SCAI).

Finally, this issue includes original articles addressing topics such as $\mathrm{PCl}$ with same-day discharge; the results of $\mathrm{PCl}$ in patients with acute coronary syndromes who are submitted to low molecular-weight and unfractionated heparin crossover; the predictors of bare-metal 
stent thrombosis; the profiles and outcomes of patients undergoing $\mathrm{PCl}$ subsidized by the Brazilian Unified Health System (Sistema Único de Saúde - SUS) or by private health care; Doppler evaluation of radial artery patency in patients using this access route for coronary procedures; the profile of diagnostic and therapeutic percutaneous procedures in congenital heart disease at
Instituto de Cardiologia do Rio Grande do Sul (Porto Alegre, RS, Brazil); and an experimental study that compares the quality of intimal hyperplasia, assessed by optical coherence tomography, of two drug-eluting stents with bioabsorbable polymers.

Áurea J. Chaves

Editor 